\title{
PREDICTION AND ANALYSIS OF CURRENT-VOLTAGE CHARACTERISTIC OF TRANSFER OF BINARY SALT IONS IN DIFFUSION LAYER
}

\section{NATALIA OLEGOVNA CHUBYR ${ }^{1}$, VITALY ALEXANDROVICH GUDZA ${ }^{2}$, MAKHAMET KHUSEEVICH URTENOV ${ }^{3} \&$ INNA VLADIMIROVNA SHKORKINA ${ }^{4}$}

${ }^{1}$ Kuban State Technological University, 2 Moskovskaya Str., Krasnodar, 350072, Russia

${ }^{2,3,4}$ Kuban State University, 149 Stavropolskaya Str., Krasnodar 350040, Russia

\section{ABSTRACT}

This work is aimed at derivation of equation of current-voltage characteristic (CVC) of nonstationary transfer of 1:1 electrolyte in depleted layer near ion exchange membrane, analysis of this equation, and determination of fundamental regularities of its variations. This was aided by construction of circuit diagram of diffusion layer/cation exchange membrane. It is demonstrated that total current in circuit is comprised of conduction current, determined by the flow of salt ions, and displacement current, which is consumed for generation and development of space charge region. The total current in circuit upon calculation of CVC varies in time as a consequence of increment of potential jump at moderate rate, thus, its variation can be considered as slow. In this case, the displacement current actually does not pass through the diffusion layer and it should not be considered upon calculation of CVC. The proposed equation of CVC is stable with regard to rounding errors, and at moderate rates of potential jump, it coincides with current-voltage characteristic for stationary case. The effect of nonstationarity at high rates of increment in potential jump has been analyzed.

KEYWORDS: Current-Voltage Characteristic, Membrane Systems, Diffusion Layer, Mathematical Model, Migration Current, Diffusion Current

Received: May 26, 2020; Accepted: Jun 16, 2020; Published: Jul 25, 2020; Paper Id.: IJMPERDJUN2020450

\section{INTRODUCTION}

Current-voltage characteristic (CVC) is an important property of transfer in electromembrane systems, since CVC is used to define limiting current, to analyze various regimes of electromembrane systems [1].

Qualitative and quantitative correspondence of theoretical CVC, predicted by mathematical models, and experimental CVC is the most important argument in favor of adequacy of the existing models. However, up till now the only method to obtain CVC is analysis of experimental data. Analysis of experimental CVC in overlimiting regime [2] using Fourier and wavelet analyses [3] and methods of dynamic chaos demonstrates complicated, nonstationary and unstable behavior of CVC. Theoretical study of such CVC behavior requires for special mathematical methods and models. Unavailability of such models does not allow revealing the interaction between the CVC behavior and properties of salt ion transfer in electromembrane systems. As a consequence, it is impossible to carry out optimization of electromembrane systems, including optimization of their geometrical and engineering specifications, aiming at transfer intensification in electromembrane systems. Equation for prediction of $\mathrm{CVC}$ in diffusion layer/cation exchange membrane is proposed. 


\section{METHODS}

\section{Mathematical Model}

Let us denote the left boundary of diffusion layer as $x=0$ (solution volume where constant concentration of electrolyte is maintained by forced convention), and the right boundary presenting the surface of cation exchange membrane as $\mathrm{x}=H$. Let us consider that the potential difference $\varphi_{d}(t)$ is preset on membrane, which increases linearly with time. The density of current passing through the system, $I(t)$, is the response to preset potential difference. However, current density is preset in experiments more frequently and the measured response is $\varphi_{d}(t)$.

The mathematical model for prediction of CVC in diffusion layer is formulated in the form of boundary problem [4]:

$$
\begin{aligned}
& \frac{\partial C_{i}}{\partial t}=-\frac{\partial j_{i}}{\partial x} \quad i=1,2 \\
& j_{i}=-\frac{F}{R T_{0}} z_{i} D_{i} C_{i} \frac{\partial \varphi}{\partial x}-D_{i} \frac{\partial C_{i}}{\partial x} \\
& \frac{\partial^{2} \varphi}{\partial x^{2}}=-\frac{F}{\varepsilon_{a}}\left(z_{1} C_{1}+z_{2} C_{2}\right) \\
& I_{c}=F\left(z_{1} j_{1}+z_{2} j_{2}\right)
\end{aligned}
$$

Where Eq. (1) is the equation of material balance; Eq. (2) is the Nernst-Planck equation for flows of sodium $i=1 \leftrightarrow \mathrm{Na}^{+}$and chlorine $i=2 \leftrightarrow C l^{-}$ions, the charge number of cations and anions are $z_{1}=1$ and $z_{2}=-1$, respectively; Eq. (3) is the Poisson equation for potential of electric field; Eq. (4) is the equation of interrelation between current density and ion flows, $\varepsilon_{a}$ is the dielectric permeability of solution, $F$ is the Faraday number, $R$ is the universal gas constant, $\varphi$ is the potential, $E=-\frac{\partial \varphi}{\partial x}$ is the intensity of electric field, $C_{i}, j_{i}, D_{i} I_{c}$ are the concentration, the flow, the diffusion coefficient of the i-th ion, the density of conduction current determined by ion flow.

The boundary conditions are comprised of the following boundary and initial conditions:

$$
\begin{aligned}
& C_{1}(t, 0)=C_{0}, C_{2}(t, 0)=C_{0}, \varphi(t, 0)=0 \\
& C_{1}(t, H)=C_{1 m},\left(\frac{\partial C_{2}}{\partial x}-\frac{F}{R T} z_{2} C_{2} E\right)(t, H)=0, \varphi_{d}(t)=\Delta \varphi=-d \cdot t \\
& C_{10}(x)=C_{0}, C_{20}(x)=C_{0}, \varphi_{0}(x)=0
\end{aligned}
$$

Where $d$ is the coefficient of increment rate of jump in time, V/s. 


\section{Circuit Diagram of Diffusion Layer/Cation Exchange Membrane}

Construction of circuit diagram depends on the aim of studies. Analysis of electromembrane systems is often based on the method of equivalent electric circuits; for instance, in [5], it was proposed to use them for analysis of impedance. In [6], the equation of impedance of three-layer system was derived, comprised of ion-exchange membrane and two adjacent diffusion layers. In these works, it was attempted to use equivalent electric circuits as a model described by electrochemical equations. In this work, the circuit diagram is used for analysis of interrelation of currents passing in the circuit and in diffusion layer/cation exchange membrane, required for the equation of CVC. Therefore, the circuit diagram is used as an analog used for considerations and not as a model.

A standard circuit diagram is usually comprised of resistance, capacitor, inductance coil, etc. The diffusion layer/cation exchange membrane, in addition to obvious properties of resistance and unipolar conductance, is also characterized by capacitance related with generation and development of space charge region. Numerical analysis of the boundary problem, Eqs. (1)-(7), demonstrates that moderate oscillations of current density cause significant oscillations of potential jump. At the same time, moderate oscillations of potential jump cause insignificant oscillations of current density. Besides, numerical analysis of the boundary problem, Eqs. (1)-(7), demonstrates the absence of resonance upon oscillation of potential jump at arbitrary frequency, that is, the absence of eigen frequency of oscillations, otherwise, the absence of inductance properties of diffusion layer/cation exchange membrane. As a consequence, the circuit diagram of diffusion layer/cation exchange membrane can be presented in the form of electric circuit comprised only of resistance and capacitor (Figure 1).

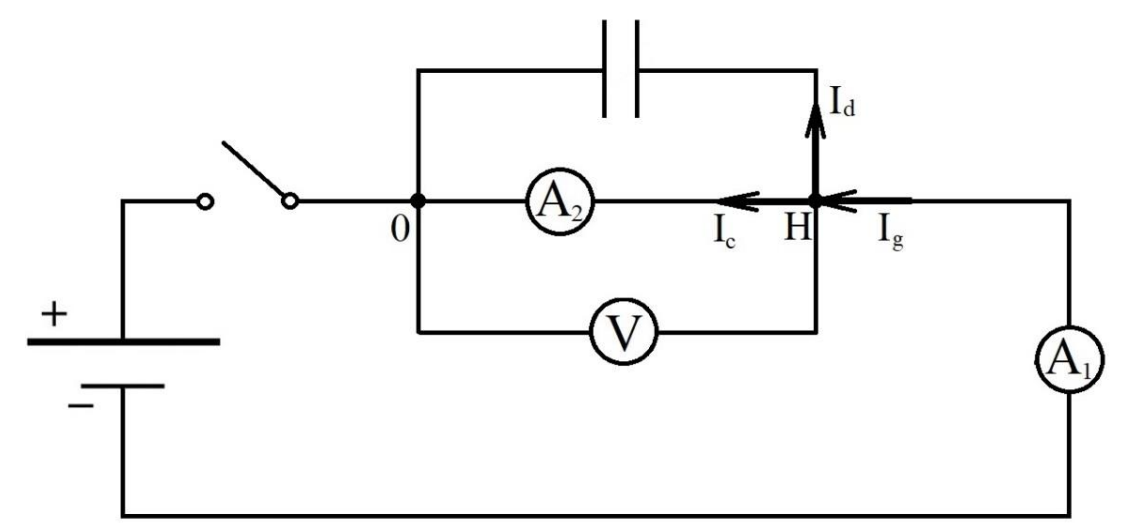

Figure 1: Electric Flowchart of Diffusion Layer: $A 1$ and $A 2$ are the Ammeters, $V$ is the Voltmeter Measuring Potential Jump between the Points $x=0$ and $x=H$.

In Figure 1 the diffusion layer/cation exchange membrane is considered as an electric circuit comprised of a certain conductor located at the segment $[0, \mathrm{H}]$ characterized by peculiar properties, related with the current determined by ion transfer, as well as a capacitor simulating accumulation of charge in the space charge region (SCR).

Let us trace the current flow in this circuit. When the circuit is closed and the current reaches the point $\mathrm{x}=\mathrm{H}$, it is splitting. A portion of electric energy, total current in the circuit $I_{g}(t)$, depending only on $t$, is consumed for salt ion transfer, that is, for current flow $I_{s}(t, x)$, stipulated by salt ion flow and depending on $t$ and $x$, the other portion is consumed for occurrence and development of SCR, that is, on flow of displacement current $I_{d}(t, x)$ depending also on $t$ 
and $\mathrm{x}$. Therefore, the point $\mathrm{x}=\mathrm{H}$ is a node, and according to the first Kirchhoff law [7] resulting from the charge conservation law, the following equality is valid:

$$
I_{g}=I_{s}+I_{d}
$$

The current $I_{d}$ can be calculated using the equations and independence of total current $I_{g}$ on $x$. It follows from Eq. (1) $[8,9]$ :

$$
\frac{\partial\left(z_{1} C_{1}+z_{2} C_{2}\right)}{\partial t}=-\frac{\partial\left(z_{1} j_{1}+z_{2} j_{2}\right)}{\partial x} \text { or } \frac{\partial \rho}{\partial t}=-\frac{\partial I_{c}}{\partial x} .
$$

It follows from Eq. (3):

$$
\varepsilon_{a} \frac{\partial E}{\partial x}=F\left(z_{1} C_{1}+z_{2} C_{2}\right)=\rho
$$

Hence,

$$
\begin{aligned}
& \mathcal{E}_{a} \frac{\partial}{\partial t} \frac{\partial E}{\partial x}=-\frac{\partial I}{\partial x} \\
& \text { or, integrating over } x \text {, we obtain: }
\end{aligned}
$$

$\varepsilon_{a} \frac{\partial E}{\partial t}=-I+\operatorname{Const}(t)$

where the constant Const $=I+\varepsilon_{a} \frac{\partial E}{\partial t}$ depends only on $t, \mathrm{~A} / \mathrm{m}^{2}$, equaling to the sum of current $I$, related with the flow of ions and displacement current $I_{d}=\varepsilon_{a} \frac{\partial E}{\partial t}$, thus, it is the total current [10], that is: $I_{g}=I_{s}+I_{d}$

\section{RESULTS}

\section{CVC Equation}

Let us determine the average current density:

$$
I_{a v}(t)=\frac{1}{H} \int_{o}^{H} I(t, x) d x
$$


Table 1: Comparison of Current Density in Different Points

\begin{tabular}{|c|c|c|c|c|c|c|c|c|}
\hline$t, \mathrm{~s}$ & 0.001 & 0.005 & \multicolumn{2}{|c|}{0.01} & 0.05 & 0.1 & 0.5 & 1 \\
\hline $\mathrm{I}(t, 0), \mathrm{A} / \mathrm{m}^{2}$ & 0.000124 & 0.000608 & \multicolumn{2}{|c|}{0.001200} & 0.005700 & 0.010963 & 0.047326 & 0.081558 \\
\hline $\mathrm{I}(t, H / 2), \mathrm{A} / \mathrm{m}^{2}$ & 0.000124 & 0.000608 & \multicolumn{2}{|c|}{0.001200} & 0.005700 & 0.010963 & 0.046536 & 0.081550 \\
\hline $\mathrm{I}(t, H), \mathrm{A} / \mathrm{m}^{2}$ & 0.000119 & 0.000885 & \multicolumn{2}{|c|}{0.002266} & 0.019974 & 0.052022 & 0.460630 & 1.018400 \\
\hline $\mathrm{I}_{\mathrm{av}}(t), \mathrm{A} / \mathrm{m}^{2}$ & 0.000123 & 0.000606 & \multicolumn{2}{|c|}{0.001197} & 0.005691 & 0.010951 & 0.046516 & 0.081519 \\
\hline $\mathrm{I}_{\mathrm{d}}(t, 0), \mathrm{A} / \mathrm{m}^{2}$ & 0.000071 & 0.000069 & \multicolumn{2}{|c|}{0.000067} & 0.000063 & 0.000059 & 0.000046 & 0.000038 \\
\hline $\mathrm{I}_{\mathrm{d}}(t, H / 2), \mathrm{A} / \mathrm{m}^{2}$ & 0.000071 & 0.000069 & \multirow{2}{*}{\multicolumn{2}{|c|}{$\begin{array}{c}0.000067 \\
0.00043\end{array}$}} & 0.000063 & 0.000059 & 0.000049 & 0.000044 \\
\hline $\mathrm{I}_{\mathrm{d}}(t, H), \mathrm{A} / \mathrm{m}^{2}$ & 0.000137 & 0.00030 & & & 0.00083 & 0.00113 & 0.00207 & 0.00249 \\
\hline$t, \mathrm{~s}$ & 1 & 5 & 10 & 15 & 20 & 25 & 30 & 35 \\
\hline $\mathrm{I}(t, 0), \mathrm{A} / \mathrm{m}^{2}$ & 0.08156 & 0.2006 & 0.2402 & 0.2605 & 0.2747 & 0.2864 & 0.2971 & 0.3072 \\
\hline $\mathrm{I}(t, H / 2), \mathrm{A} / \mathrm{m}^{2}$ & 0.08155 & 0.2005 & 0.24 & 0.2603 & 0.2745 & 0.2862 & 0.2969 & 0.307 \\
\hline $\mathrm{I}(t, H), \mathrm{A} / \mathrm{m}^{2}$ & 1.01840 & 0.2023 & 0.2462 & 0.2676 & 0.2825 & 0.2947 & 0.3057 & 0.3161 \\
\hline $\mathrm{I}_{\mathrm{av}}(t), \mathrm{A} / \mathrm{m}^{2}$ & 0.08152 & 0.2005 & 0.24 & 0.2602 & 0.2744 & 0.2861 & 0.2969 & 0.3069 \\
\hline $\mathrm{I}_{\mathrm{d}}(t, 0), \mathrm{A} / \mathrm{m}^{2}$ & 0.00004 & 0.00001 & 0.0000048 & 0.0000027 & 0.000002 & 0.0000017 & 0.0000016 & 0.0000015 \\
\hline $\mathrm{I}_{\mathrm{d}}(t, H / 2), \mathrm{A} / \mathrm{m}^{2}$ & 0.00005 & 0.00003 & 0.000018 & 0.000012 & 0.0000098 & 0.0000092 & 0.0000092 & 0.0000097 \\
\hline $\mathrm{I}_{\mathrm{d}}(t, H), \mathrm{A} / \mathrm{m}^{2}$ & 0.0025 & 0.0017 & 0.00066 & 0.000329 & 0.000206 & 0.00016 & 0.00014 & 0.00013 \\
\hline
\end{tabular}

It follows from Table 1 that the displacement current is sufficiently high only at short times (up to $0.5 \mathrm{~s}$ in Table 1 ).

Though the total current is not constant in time, its variation upon prediction of CVC at moderate increment rate $d$ of potential jump can be considered as slow. In this case, the capacitor actually does not pass current, and CVC can be predicted without consideration for the displacement current, as follows from Table 1. In addition, while predicting CVC, the average current density $I_{a v}(t)$ can be used, which is stable with regard to rounding errors.

Let us compare the CVC for nonstationary case with moderate rate $d$ and the CVC for stationary problem.

Table 2: Potential Jump at Various Current Densities. Line 2: Calculations by Eq. (8) for Nonstationary Problem at $\mathrm{d}=\mathbf{0 . 0 1}$, Line 3: for Stationary Problem

\begin{tabular}{|c|c|c|c|c|c|c|c|}
\hline$\Delta \varphi, \mathrm{V}$ & 0.13 & 0.23 & 0.24 & 0.25 & 0.51 & 0.79 & 1.05 \\
\hline$\frac{I_{s}}{I_{\text {cond }}}$ & 0,85 & 0,98 & 0,99 & 1 & 1.2 & 1.4 & 1.6 \\
\hline$\frac{I_{s}}{I_{\text {cond }}}$ & 0.8 & 0.91 & 0.92 & 0.93 & 1.1 & 1.27 & 1.43 \\
\hline
\end{tabular}

As can be seen in Table 2, the CVC for nonstationary and stationary problems is close at moderate increment rates of potential jump.

\section{CVC Analysis}

Using Eqs. (1)-(4), it is possible to write as follows [11]: $I_{c}=I_{m i g}+I_{\text {diff }}$,

where $I_{\text {mig }}=\frac{F^{2}}{R T_{0}}\left(z_{1}^{2} D_{1} C_{1}+z_{2}^{2} D_{2} C_{2}\right) E$ is the migration current, a portion of total conduction current $\mathrm{I}_{\mathrm{c}}$, stipulated by potential jump imposed on the system, and $I_{\text {diff }}=-\frac{F \cdot \partial\left(z_{1} D_{1} C_{1}+z_{2} D_{2} C_{2}\right)}{\partial x}$ is the diffusion current, a portion of total conduction current $I_{c}$, which is stipulated by existence of diffusion potential $\psi(t, x)=z_{1} D_{1} C_{1}+z_{2} D_{2} C_{2}$. 
Let us determine averaged migration and diffusion constituents of current:

$$
\begin{aligned}
& I_{\text {mig,av }}(t)=\frac{F^{2}}{R T_{0} H} \int_{0}^{H}\left(z_{1}^{2} D_{1} C_{1}+z_{2}^{2} D_{2} C_{2}\right) E(t, x) d x \\
& I_{\text {diff ,av }}(t)=-F \frac{z_{1} D_{1} C_{1}(t, H)-z_{1} D_{1} C_{1}(t, 0)+z_{2} D_{2} C_{2}(t, H)-z_{2} D_{2} C_{2}(t, 0)}{H}
\end{aligned}
$$
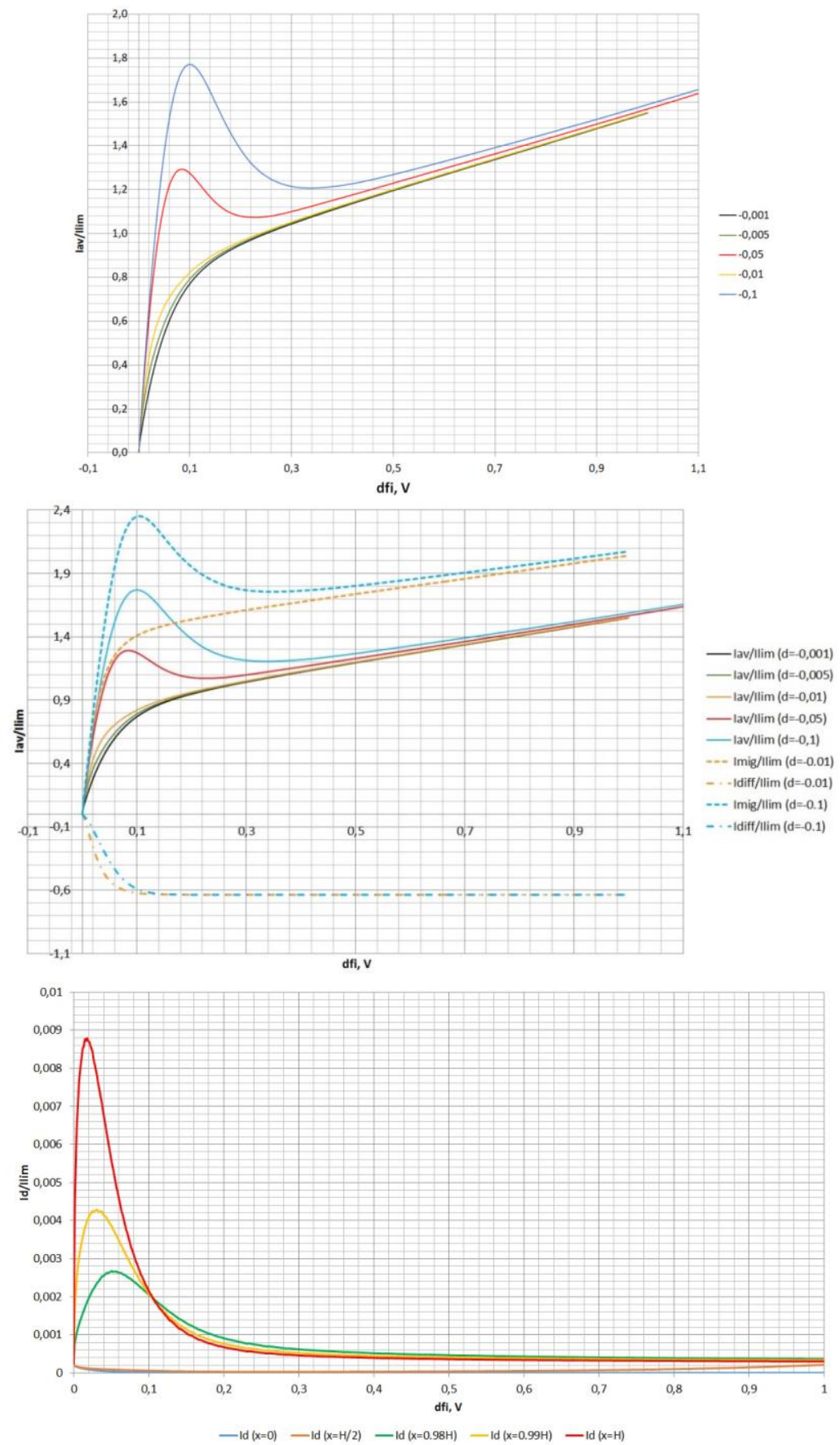

Figure 2: CVC Plots. Ordinate: Relative Current Density $i_{a v}(t) / i_{n p}$, Abscissa: Potential Jump, V: a) As a

Function of Increment Rate of Potential Jump; b) Comparison of Average Current with Average Migration and Diffusion Currents; c) Cross Section of Displacement Current Plot $I_{d, a v}(t, x)$ at Various $x$ 
Comparison of the acquired results with calculations and analysis of CVC as a function of scanning rate of current/potential in [10] shows their agreement with high accuracy.

It can be seen in Fig. 2a) that at low $d$, starting from $0.01, \mathrm{CVC}$ actually does not depend on $d$. Herewith, CVC values are close to current density determined by stationary model for respective potential jumps, thus, it is possible to consider that quasi-stationary regime takes place. At high $d$, near $t=0$, there are splashes, which are sufficiently quickly faded, and CVC reaches quasi-stationary regime (the splash and the time of reaching quasi-stationary regime depend on $d$ ). The splash is related with nonstationarity at high $d$ [12].

It can be seen in Fig. 2b) that in sublimiting regime, the migration current and the diffusion current give approximately equal contribution to total current, and in superlimiting current, the main contribution is made by the migration current (about $75 \%$ of total current).

Let us determine the averaged displacement current $I_{d, a v}(t)=\frac{\varepsilon_{r}}{H} \int_{0}^{H} \frac{\partial E(t, x)}{\partial t} d x$.

Upon linear increment of potential jump $\varphi_{d}(t)=-d \cdot t$, we have as follows:

$$
\begin{aligned}
& I_{d, a v}(t)=\frac{\varepsilon_{r}}{H} \int_{0}^{H} \frac{\partial E(t, x)}{\partial t} d x=-\frac{\varepsilon_{r}}{H} \frac{\partial \varphi(t, H)}{\partial t}=\frac{\varepsilon_{r} d}{H} \\
& I_{d, a v}(t)=\frac{\varepsilon_{r} d}{H} \text { does not depend on } t \text { and demonstrates that the higher is the scanning rate, the higher is the }
\end{aligned}
$$
displacement current, moreover, starting from several seconds, it is significantly lower than the average current $\mathrm{I}_{\mathrm{av}}(t)$. For instance, $I_{d, a v}=7 \cdot 10^{-5} \mathrm{~A} / \mathrm{m}^{2}$ or $\frac{I_{d, a v}}{I_{\text {lim }}} \approx 0.23 \cdot 10^{-3}$ at $d=0.01 \mathrm{~V} / \mathrm{s}$.

\section{CONCLUSIONS}

This work proposes circuit diagram for nonstationary transfer of 1:1 electrolyte in depleted layer near cation exchange membrane, which simplifies analysis of currents, and equation of CVC, stable with regard to rounding errors. The interrelations between total current and conducting and displacement currents have been analyzed. It is demonstrated that in sublimiting regime, the migration current and diffusion current provide approximately equal contribution to total current, and in over limiting regime, the main contribution is made by the migration current.

\section{ACKNOWLEDGMENTS}

This work was supported by the Russian Foundation for Basic Research, project No. 19-08-00252 A: Theoretical and experimental studies of current-voltage characteristic of electromembrane systems.

\section{REFERENCES}

1. Zabolotskii, V.I., Nikonenko, V.V. Perenos ionov v membranakh [Ion transfer in membranes]. Nauka, Moscow, 1996. 
2. Budnikov, E.Yu. Analysis of fluctuation phenomena in the range of superlimiting currents in electromembrane system: Cand. thesis. State Scientific Center of the Russian Federation, Research Institute of Physico-Chemical Institute named after L.Ya. Karpov, Moscow, 2000.

3. Korotkova, Tatyana Germanovna, Aleksandra Sergeevna Danilchenko, and Yuriy Nikolaevich Sedoy. "Evaporation Rate of Water from Glass Surface under Natural and Forced Convection." International Journal of Mechanical and Production Engineering Research and Development 9.4 (2019): 955-962.

4. Budnikov, E.Yu., Kukoev, I.Yu., Maksimychev, A.V., Miroshnikova, I.N., Timashev, S.F., Gulyaev, A.M. Veivlet i Fur'e-analiz elektricheskikh fluktuatsii v poluprovodnikovykh i elektrokhimicheskikh sistemakh [Wavelet and Fourier analysis of electric fluctuations in semiconductor and electrochemical systems]. Izmeritel'naya Tekhnika 11 (1999) 40-44.

5. Chubyr', N.O., Kovalenko, A.V., Urtenov, M.Kh. Chislennye i asimptoticheskie metody analiza perenosa 1:1 elektrolita v membrannykh sistemakh [Numerical and asymptotic methods of analysis of transfer of 1:1 electrolyte in membranes]. Kuban State University, Krasnodar, 2018.

6. Macdonald, D.D. Transient Techniques in Electrochemistry. Plenum Press, New York, 1977.

7. Gandhi, Ronak, Gajendra Patel, and Harshkumar Sharma. "Simulation of Distributed Power Flow Controller Facts Device in Voltage Sag and Swell Mitigation." International Journal of Electrical and Electronics Engineering Research (IJEEER) 7. 2, Apr 2017, 39-44

8. Nikonenko, V.V., Kozmai, A.E. Electrical equivalent circuit of an ion-exchange membrane system. Electrochimica Acta 56 (2011) 1262-1269.

9. Sivukhin, D.V. Obshchii kurs fiziki [General course in physics]. Vol. III. Electricity. 4th edition. FIZMATLIT; MFTI, Moscow, 2004.

10. Uzdenova, A., Kovalenko, A., Urtenov, M., Nikonenko, V. $1 D$ mathematical modelling of non-stationary ion transfer in the diffusion layer adjacent to an ion-exchange membrane in galvanostatic mode. Membranes 8(3) (2018) 84.

11. Srikanth, Ks, Lv Narasimha Rao, and Vaddi Ramesh. "Improvement of Microturbine Generator Output Voltage Using Matrix Converter." International Journal of Electrical and Electronics Engineering Research (IJEEER) 4. 2, Apr 2014, 319 -326

12. Urtenov, M.Kh., Kovalenko, A.V., Sukhinov, A.I., Chubyr, N.O., Gudza, V.A. Model and numerical experiment for calculating the theoretical current-voltage characteristic in electro-membrane systems. IOP Conference Series: Materials Science and Engineering 680(1) (2019) 012030.

13. Manzanares, J.A., Murphy, W.D., Mafe, S., Reiss, H. Numerical Simulation of the Nonequilibrium Diffuse Double Layer in Ion-Exchange Membranes. J. Phys. Chem. 97 (1993) 8524-8530.

14. HA, P. Anus, and K. Swarnasri. "A Novel Approach to Compensate Voltage Sag in Three Phase Lines." International Journal of Electrical and Electronics Engineering Research (IJEEER) 4. 2, Apr 2014, 229-238

15. Newman, J. Electrochemical Systems. Prentice-Hall, Inc., Engelewood Cliffs, N.J., 1973.

16. Moya, A.A., Sistat, P. Reaching the limiting current regime by linear sweep voltammetry in ion-exchange membrane systems. Journal of Membrane Science 555 (2018) 134-145. 